\title{
PRODUCT AND MARKOV MEASURES OF TYPE III
}

\author{
ANTHONY H. DOOLEY, IVO KLEMEŠ and ANTHONY N. QUAS
}

(Received 25 May 1997; revised 25 March 1998)

Communicated by J. R. J. Groves

\begin{abstract}
We give some explicit constructions of type III product measures with various properties, resolving some conjectures of Brown, Dooley and Lake. We also define a family of Markov odometers of type $\mathrm{III}_{0}$ and show that the associated flow is approximately transitive.
\end{abstract}

1991 Mathematics subject classification (Amer. Math. Soc.): primary 28D15.

Keywords and phrases: AT property, $G$-measures, orbit equivalence, non-singular transformations.

\section{Introduction}

Dye's celebrated theorem [8] states that any ergodic non-singular action of a countable amenable group is orbit equivalent to a measured odometer action. Hence, a complete classification of these amenable group actions up to orbit equivalence will follow from a classification up to orbit equivalence of measured odometer actions.

There is a well-known classification of ergodic non-singular group actions into classes I, II and III respectively according to whether the measure is concentrated on a single orbit, the measure is equivalent to an invariant measure (finite or $\sigma$-finite) or neither of the above holds. The case I is a relatively trivial case. The case II is fairly well understood, so the remaining interesting case (which in some sense is the most prevalent) is case III.

Krieger introduced a subdivision of case III using an invariant which he called the ratio set (see [13] and [14]). The ratio set may be informally defined as the set of limits of ratios $d \mu \circ \gamma / d \mu$ for $\gamma$ in the group $\Gamma$ of transformations and it may be shown to be a closed subset of $\mathbb{R}^{+} \cup\{0, \infty\}$ which is closed under multiplication. There are then three possibilities for the ratio set. It can be $\{0,1, \infty\}, \mathbb{R}$ or $\left\{\lambda^{n}: n \in \mathbb{Z}\right\}$ for 
a fixed $\lambda \in(0,1)$. The corresponding classes of transformations are known as $\mathrm{III}_{0}$, $\mathrm{III}_{1}$ and $\mathrm{III}_{\lambda}$. It may be shown that any two systems in $\mathrm{III}_{1}$ are orbit equivalent (see [13]). Similarly for any fixed $\lambda \in(0,1)$, any two $\mathrm{III}_{\lambda}$ systems are orbit equivalent. The situation for $\mathrm{III}_{0}$ is much less well understood and it is this category upon which we shall focus.

We will, as described above, be considering measured odometer actions. A natural class of examples is given by the actions where the measure is a product measure. An action which is orbit equivalent to one of this type is said to be product type. A necessary and sufficient condition for an odometer action to be of product type was introduced by Connes and Woods [5] who use a proof based on operator algebras. The condition which they introduced is that the Poincare flow associated to the action has a property which they call approximately transitive or AT. Hawkins [12] showed necessity of Connes and Woods' condition with a simpler ergodic theoretic proof and Hamachi [10] was able to show sufficiency, by using purely ergodic techniques.

It nevertheless remains a difficult task to give examples of measures of type $\mathrm{II}_{0}$ or, given a product measure to decide whether it is of type $\mathrm{III}_{0}$. (Moore's criterion [16] allows us readily to decide when a product measure is of type $\mathrm{I}_{1} \mathrm{II}_{1}, \mathrm{II}_{\infty}$ or III.)

Hamachi, Oka and Osikawa [11] produced examples of product measures of type $\mathrm{III}_{0}$ and Krieger [15] gave an example of a non-AT action.

Brown and Dooley [2] introduced the notion of a $G$-measure. (Their formalism favoured the use of the groups of finite coordinate changes over the odometer; these two actions have the same orbits.) These provide an explicit description of in some senses the most general quasi-invariant measure. In [3], it was shown how to compute the ratio sets of $G$-measures in some cases, and the machinery was applied to product measures. However, there were some unresolved conjectures and some rather sketchy proofs.

In this paper, we aim to refine the techniques of [3], resolve some conjectures therein and give full details of some results on product measures. At the same time, we are able to somewhat sharpen the examples of type $\mathrm{III}_{0}$ product measures in [11]. From the perspective of $G$-measures, the next most complicated measures after product measures are Markov measures. A second aim is to consider a class of Markov measures on the infinite product of two point spaces, where the transition probabilities remain constant on long blocks. We are able to explicitly compute the Poincare flow of such a measure and show that it is AT.

A detailed description of the results follows. Consider the infinite product measure $\mu=\otimes \mu_{i}$ on $X=\prod \mathbb{Z}_{2}$, where $\mu_{i}(\{0\})=\frac{1}{2}\left(1+a_{i}\right), \mu_{i}(\{1\})=\frac{1}{2}\left(1-a_{i}\right) \quad(-1<$ $a_{i}<1$ ). In section 2 , we give an example of a measure of type III, but not of type III with $a_{i} \nearrow 1$, disproving a conjecture in [3]. We also give a relatively easy example of a family of product measures of type $\mathrm{III}_{0}$ on $X$. The example itself is similar to one found in [10], but we give a short and direct proof that the ratio set is $\{0,1, \infty\}$. 
Examples are found by taking a suitable sequence $\left\{a_{i}\right\}$ which is constant on blocks of increasing length. In section 3 , we give a detailed proof that if $a_{i} \rightarrow 0$ and $\sum a_{i}^{2}=\infty$ then $\mu$ is of type III $_{1}$, providing full details of a claim made in [3]. The essential technique in these two sections is Lemma 2.1, a generalization of [3, Theorem 3.1] and a primitive version of [10, Theorem 1].

The final section considers Markov measures on the infinite product of two point spaces, which have the property that their transition probabilities are constant on long blocks, behaving in the same way as the probabilities in the examples of section 2 . We are able to compute the Poincare flow explicitly as an odometer with parity bit. These flows are AT, and hence the measures are orbit equivalent to product measures (although they are certainly far from being equivalent to products).

More recent work of Dooley and Hamachi [7] finds examples of non-AT Markov measures. These are realized on $\prod \mathbb{Z}_{l(n)}$ where $l(n)$ increases rapidly.

\section{Definitions and notation}

We consider transformations of finite or $\sigma$-finite measure spaces. The transformations which we consider will be measurable and invertible, with measurable inverses and be non-singular: that is a set has measure 0 if and only if its image has measure 0 . These transformations will be known as isomorphisms. In the case where the transformation is from a measure space $X$ to itself, it will be called an automorphism of $X$. $\Gamma$ will denote a countable group of automorphisms of $(X, \mathscr{B}, \mu)$. The full group $[\Gamma]$ of $\Gamma$ consists of those automorphisms $\theta$ of $X$ which have the property that for almost every $x \in X, \theta(x)=\gamma(x)$ for some $\gamma \in \Gamma$. Note that we use similar notation for the orbit of a point. Namely, $[x]$ is the orbit of the point $x$ under the group $\Gamma$ of transformations.

As an example, define $X$ to be $\{0,1\}^{\mathbb{Z}^{+}}$and $\Gamma$ to be the group generated by the maps $\gamma_{n}$ which reverses the $n$th coordinate ( $\left.\operatorname{so}\left(\gamma_{n}(x)\right)_{i}=\delta_{i n}+x_{i} \bmod 2\right)$. Then defining $\theta$ to be the standard odometer mapping obtained by regarding points of $x$ as 2-adic integers and adding 1 (with carry) (so $\theta(\ldots 10110)=\ldots 10111 ; \theta(\ldots 10111)=\ldots 11000)$, we see that $\theta \in[\Gamma]$. In this context, let $\Gamma_{n}$ (respectively $X_{n}$ ) be the elements of $\Gamma$ (respectively $X$ ) whose coordinates after the $n$th are zero and let $\Gamma^{n}$ (respectively $X^{n}$ ) be those elements whose first $n$ coordinates are zero.

Two group actions $\Gamma$ acting on a measure space $\left(X_{1}, \mathscr{B}_{1}, \mu_{1}\right)$ and $\Gamma^{\prime}$ acting on $\left(X_{2}, \mathscr{B}_{2}, \mu_{2}\right)$ are orbit equivalent (sometimes also called weakly equivalent) if there exists an isomorphism $\Phi$ from $X_{1}$ to $X_{2}$ such that for almost every $x \in X_{1}, \Phi([x])=$ $[\Phi(x)]$. In the example above, the actions of $\Gamma$ on $X$ and $\left\{\theta^{n}: n \in \mathbb{Z}\right\}$ on $X$ are orbit equivalent.

The ratio set $R_{\mu}=r(X, \Gamma, \mu)$, as defined in [14], is the set of $r$ in $[0, \infty]$ such 
that for each $\epsilon>0$ and set $A$ of positive measure, there exists a subset $B \subset A$ of positive measure and a $\theta \in[\Gamma]$ such that $\theta(B) \subset A$ and $|d \mu \circ \theta / d \mu-r|<\epsilon$ on $B$. In our case, where $\Gamma$ is a countable group, it is equivalent to define $r \in R$ if and only if for each $\epsilon>0$ and set $A$ of positive measure, there exists a subset $B$ of positive measure and $\gamma \in \Gamma$ such that $\gamma(B) \subset A$ and $|d \mu \circ \gamma / d \mu-r|<\epsilon$ on $B$ (that is the automorphism $\theta$ may be chosen from the group itself, not the full group). We use this latter definition in what follows.

Given an action of a group $\Gamma$ on a space $X$, we define an action of $\Gamma$ on $X \times \mathbb{R}$. For $\gamma \in \Gamma$, define $\tilde{\gamma}(x, t)=(\gamma(x), t-\log (d \mu \circ \gamma / d \mu(x)))$. Then form $Y=X \times \mathbb{R} / \Gamma$, the set of $\Gamma$-orbits in $X \times \mathbb{R}$. There is a natural projection $\pi$ from $X \times \mathbb{R}$ to $Y$. The measure on $Y$ is taken to be the projection of $\mu \times v$ where $d \nu(x)=\exp x d \lambda(x)$. The projection can be used to give $Y$ a $\sigma$-algebra by defining a subset to be measurable if and only if its inverse image under $\pi$ is a measurable subset of $X$. Since the action of $\mathbb{R}$ on $X \times \mathbb{R}$ given by $\theta_{s}(x, t)=(x, s+t)$ commutes with the action of $\Gamma$ on $X \times \mathbb{R}$, it follows that the action of $\mathbb{R}$ may be pushed down to an action on $Y$. This is the associated flow (or Poincare flow) of the action of $\Gamma$ on $X$.

An important property which the associated flow may or may not possess is approximate transitivity (abbreviated to the AT property, so we often say if this property holds that the associated flow is AT). An action of a group $G$ on a measure space $X$ is AT if for all $\epsilon>0$ and any sequence $f_{1}, f_{2}, \ldots, f_{n}$ of functions in $L^{1}(X)^{+}$, the space of positive integrable functions, there exists a function $f \in L^{1}(X)^{+}$, finitely many elements $g_{i, j}$ of $G$ and constants $\lambda_{i, j}$ such that

$$
\left\|f_{i}-\sum_{j}\left(\lambda_{i . j} f \circ g_{i, j} \frac{d \mu \circ g_{i . j}}{d \mu}\right)\right\|_{1}<\epsilon
$$

for each $i$. We will write $\mathscr{L}_{g}(f)$ for the function defined by

$$
\mathscr{L}_{g}(f)(x)=f(g(x)) \frac{d \mu \circ g}{d \mu}(x) .
$$

Note that for positive functions $f, \mathscr{L}_{g}$ is an $L^{\prime}$ norm-preserving operator. The AT condition may be re-expressed as $\left\|f_{i}-\sum_{j} \lambda_{i, j} \mathscr{L}_{g_{i, j}} f\right\|_{1}<\epsilon$.

\section{Product measures of type $\mathrm{III}_{0}$}

EXAMPLE 1. In the notation of [3], we give an example with $a_{i} \nearrow 1$ such that the ratio set is not a subset of $\{0,1, \infty\}$. This disproves [3, Conjecture 6.3].

Fix $\rho>0$. Let $\left\{\sigma_{i}\right\}$ be a sequence of the form

$$
\rho, \rho, \ldots, \rho, 2 \rho, 2 \rho, \ldots, 2 \rho, 3 \rho, \ldots
$$


such that if $n_{k}$ denotes the number of terms of type $k \rho$, then

$$
n_{k} \geq e^{k \rho} \text { and } n_{k+1} \geq n_{k} .
$$

The $\left\{a_{i}\right\}$ are then determined as in [3] by

$$
\frac{1+a_{i}}{1-a_{i}}=e^{\sigma_{i}}, \quad \text { so } \quad a_{i}=\frac{e^{\sigma_{i}}-1}{e^{\sigma_{i}}+1} \nearrow 1 .
$$

Let $\mu$ denote the resulting measure. We prove:

PROPOSITION 2.1. $e^{-\rho} \in R_{\mu}$.

We need the following sufficient condition. It is a generalization of [3, Theorem (3.2) (i)] to the case of more than one $\gamma \in \Gamma^{n}$. In this case, we need to assume the disjointness of the sets of $u$ 's for these $\gamma$ 's, and that of their images under the $\gamma$ 's:

LEMMA 2.1. Let $r \in(0, \infty)$. Suppose that $\forall \epsilon>0, \exists \beta>0$ such that $\forall n, \forall \gamma_{0} \epsilon$ $\Gamma_{n}$ there exists an integer $K$ and there exist

$$
\gamma_{1}, \gamma_{2}, \ldots, \gamma_{K} \in \Gamma^{n} \text { and } \mathscr{U}_{1}, \mathscr{U}_{2}, \ldots, \mathscr{U}_{K} \subset \gamma_{0} X^{n}
$$

such that:

(i) the $\left\{\mathscr{U}_{j}\right\}$ are disjoint,

(ii) the $\left\{\gamma_{j} \mathscr{U}_{j}\right\}$ are disjoint,

(iii) $\mu\left(\cup_{j=1}^{K} \mathscr{U}_{j}\right) \geq \beta \mu\left(\gamma_{0} X^{n}\right)$,

(iv) $\forall j, u \in \mathscr{U}_{j} \Rightarrow\left|\left(\left(d \mu \circ \gamma_{j}\right)(u) / d \mu\right)-r\right|<\epsilon$;

then $r \in r(X, \Gamma, \mu)$.

PROOF OF LEMMA 2.1. The proof is based on the method of proof of $[3$, Theorem 3.2(i)].

Let $r>0$ and let $\epsilon<r$ (this will be specified). Let $\beta>0$ be fixed as in the statement. Let $A$ be an arbitrary set of positive $\mu$-measure. Then there exists $n$ and $\gamma_{0} \in \Gamma_{n}$ such that

$$
\mu\left(A \cap \gamma_{0} X^{n}\right)>(1-c) \mu\left(\gamma_{0} X^{n}\right)
$$

where $c$ is to be specified.

For this $n$ and $\gamma_{0}$, there exist $\left\{\gamma_{j}\right\}$ and $\left\{\mathscr{U}_{j}\right\}$ as stated. It is given that $\bigcup \mathscr{U}_{j}$ fills up a proportion $\beta$ of $\gamma_{0} X^{n}$. We check that $\bigcup \gamma_{j} \mathscr{U}_{j}$ also has this property for some constant $\beta^{\prime}$.

Since $\left|\left(\left(d \mu \circ \gamma_{j}\right)(u) / d \mu\right)-r\right|<\epsilon$ on $\mathscr{U}_{j}$, it follows that for all $j, \mu\left(\gamma_{j} \mathscr{U}_{j}\right) \geq$ $(r-\epsilon) \mu\left(\mathscr{U}_{j}\right)$. So by disjointness,

$$
\mu\left(\bigcup \gamma_{j} \mathscr{U}_{j}\right) \geq(r-\epsilon) \mu\left(\bigcup \mathscr{U}_{j}\right) \geq(r-\epsilon) \beta \mu\left(\gamma_{0} X^{n}\right) .
$$


Thus we can take $\beta^{\prime}=(r-\epsilon) \beta$.

We now claim that for at least one index $j=j_{0}$, we have

$$
\mu\left(A \cap\left[\gamma_{j_{0}}\left(A \cap \mathscr{U}_{j_{0}}\right)\right]\right)>0 .
$$

We have $\mu\left(A \cap\left(\bigcup \mathscr{U}_{j}\right)\right) \geq \mu\left(\bigcup \mathscr{U}_{j}\right)-\mu\left(\left[\gamma_{0} X^{n}\right] \backslash A\right) \geq(\beta-c) \mu\left(\gamma_{0} X^{n}\right)$.

Hence, using disjointness:

$$
\begin{aligned}
\mu\left(\bigcup_{j} \gamma_{j}\left(A \cap \mathscr{U}_{j}\right)\right) & =\sum_{j} \mu\left(\gamma_{j}\left(A \cap \mathscr{U}_{j}\right)\right) \geq \sum_{j}(r-\epsilon) \mu\left(A \cap \mathscr{U}_{j}\right) \\
& =(r-\epsilon) \mu\left(\bigcup\left(A \cap \mathscr{U}_{j}\right)\right) \geq(r-\epsilon)(\beta-c) \mu\left(\gamma_{0} X^{n}\right) .
\end{aligned}
$$

By definition, the set $S=\bigcup_{j} \gamma_{j}\left(A \cap \mathscr{U}_{j}\right)$ is contained in $\gamma_{0} X^{n}$ and we have shown that

$$
\mu(S) \geq(r-\epsilon)(\beta-c) \mu\left(\gamma_{0} X^{n}\right) .
$$

Hence

$$
\mu(A \cap S) \geq \mu(S)-\mu\left(\left[\gamma_{0} X^{n}\right] \backslash A\right) \geq((r-\epsilon)(\beta-c)-c) \mu\left(\gamma_{0} X^{n}\right) .
$$

This is positive if we ensure that $\epsilon<r$ and $c<\min (\beta / 2,(r-\epsilon) \beta / 2)$.

But the statement $\mu(A \cap S)>0$ gives

$0<\mu\left(A \cap \bigcup_{j} \gamma_{j}\left(A \cap \mathscr{U}_{j}\right)\right)=\sum_{j} \mu\left(A \cap\left[\gamma_{j}\left(A \cap \mathscr{U}_{j}\right)\right]\right) \quad$ by disjointness of $\gamma_{j} \mathscr{U}_{j}$.

Hence there exists $j=j_{0}$ with

$$
\mu\left(A \cap\left[\gamma_{j_{0}}\left(A \cap \mathscr{U}_{j_{0}}\right)\right]\right)>0 .
$$

It follows that letting

$$
B=\left\{a \in A \cap \mathscr{U}_{j_{0}}: \gamma_{j_{0}} a \in A\right\} \subset A
$$

we get $\gamma_{j_{0}} B=A \cap\left[\gamma_{j_{0}}\left(A \cap \mathscr{U}_{j_{0}}\right)\right] \subset A$ and $\mu\left(\gamma_{j_{0}} B\right)>0$, which also implies $\mu(B)>0$.

Also, since $B \subset \mathscr{U}_{j_{0}}$, we have

$$
\left|\frac{d \mu \circ \gamma_{j_{0}}}{d \mu}(u)-r\right|<\epsilon \quad \forall u \in B .
$$

Since $\epsilon>0$ was arbitrary, we have $r \in r(X, \tau, \mu)$. 
PROOF OF PROPOSITION 2.1. We shall in effect take $\epsilon=0$ and find some $\beta>0$. Let any $n, \gamma_{0} \in \Gamma_{n}$ be given. Then, in $\left\{\sigma_{i}\right\}_{i=n}^{\infty}$ there is a block of the form

$$
\sigma_{i}=\rho k, \ldots, \rho k, \rho(k+1), \rho(k+1), \ldots
$$

for some $k$. Fix such a block and $k$. Since $n_{k+1} \geq n_{k}$, there are available at least $n_{k}$ terms of the next constant, $\rho(k+1)$, to the right of this block.

The following table defines our choices of $\left\{\gamma_{j}\right\}$ and $\left\{\mathscr{U}_{j}\right\}\left(K=n_{k}\right)$ (below $\left.K=4\right)$

$$
\begin{aligned}
& \sigma_{i} \quad=\ldots, \rho k, \rho k \rho k \rho k \rho(k+1), \rho(k+1), \rho(k+1), \rho(k+1) \ldots \\
& \left(\gamma_{1}\right)_{i}=\begin{array}{lllllllllllll}
0 & 1 & 0 & 0 & 0 & 1 & 0 & 0 & 0 & 0 & \ldots
\end{array} \\
& \left(\gamma_{2}\right)_{i}=\begin{array}{llllllllllll}
0 & 0 & 1 & 0 & 0 & 0 & 1 & 0 & 0 & 0 & \ldots
\end{array} \\
& \left(\gamma_{3}\right)_{i}=\begin{array}{lllllllllllll}
0 & 0 & 0 & 1 & 0 & 0 & 0 & 1 & 0 & 0 & \ldots
\end{array} \\
& \left(\gamma_{K}\right)_{i}=\begin{array}{llllllllllll}
0 & 0 & 0 & 0 & 1 & 0 & 0 & 0 & 1 & 0 & \ldots
\end{array}
\end{aligned}
$$

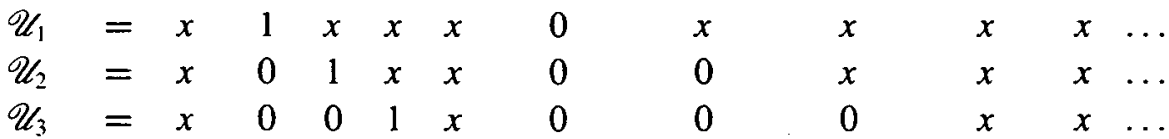

Here the convention is that $\mathscr{U}_{j}$ consists of all $u$ having the coordinates shown, and $x$ denotes either 0 or 1 . (And we assume all this in $\gamma_{0} X^{n}$.)

Clearly, applying the $\gamma_{j}$ 's to the $\mathscr{U}_{j}$ 's gives

$$
\begin{array}{llllllllllll}
\gamma_{1} \mathscr{U}_{1} & = & x & 0 & x & x & x & 1 & x & x & x & x \\
\gamma_{2} \mathscr{U}_{2} & = & x & 0 & 0 & x & x & 0 & 1 & x & x & x \\
\gamma_{3} \mathscr{U}_{3} & = & x & 0 & 0 & 0 & x & 0 & 0 & 1 & x & x \\
\gamma_{4} \mathscr{U}_{4} & = & x & 0 & 0 & 0 & 0 & 0 & 0 & 0 & 1 & x
\end{array}
$$

The disjointness of the $\left\{\mathscr{U}_{j}\right\}_{j=1}^{n_{k}}$ is guaranteed by the 1's on the diagonal, preceded by the 0 's. Similarly for the $\left\{\gamma_{j} \mathscr{U}_{j}\right\}$.

Next, let us verify that

$$
u \in \mathscr{U}_{j} \Longrightarrow \frac{d \mu \circ \gamma_{j}}{d \mu}(u)=e^{-\rho}
$$

[We are following the convention

$$
\left.\mu_{i}(\{0\})=\frac{1+a_{i}}{2}=\frac{1}{1+e^{-\sigma_{i}}}:=1-p_{i}:=q_{i}, \mu_{i}(\{1\})=\frac{1-a_{i}}{2}=\frac{e^{-\sigma_{i}}}{1+e^{-\sigma_{i}}}:=p_{i} .\right]
$$


Thus if $u \in \mathscr{U}_{j}$ then

$$
\frac{d \mu \circ \gamma_{j}}{d \mu}(u)=\frac{\mu_{(1)}(0) \mu_{(2)}(1)}{\mu_{(1)}(1) \mu_{(2)}(0)}=\frac{1}{e^{-\sigma_{(1)}}} \frac{e^{-\sigma_{(2)}}}{1}=e^{\sigma_{(1)}-\sigma_{(2)}}=e^{k \rho-(k+1) \rho}=e^{-\rho}
$$

where $\sigma_{(1)}, \sigma_{(2)}$ denote $k \rho$ and $(k+1) \rho$ for convenience.

It remains to estimate the measures $\mu\left(\mathscr{U}_{j}\right) / \mu\left(\gamma_{0} X^{n}\right)$. We get

$$
\begin{aligned}
& \tilde{\mu}\left(\mathscr{U}_{1}\right)=p_{(1)} q_{(2)} \geq p_{(1)} q_{(1)}, \\
& \tilde{\mu}\left(\mathscr{U}_{2}\right)=q_{(1)} p_{(1)} q_{(2)}^{2} \geq p_{(1)} q_{(1)}^{3}, \\
& \tilde{\mu}\left(\mathscr{U}_{3}\right)=q_{(1)}^{2} p_{(1)} q_{(2)}^{3} \geq p_{(1)} q_{(1)}^{5}, \text { and so on, }
\end{aligned}
$$

where $\tilde{\mu}$ denotes $\mu / \mu\left(\gamma_{0} X^{n}\right)$. Thus, using the notation $p_{(1)}=e^{-k \rho} /\left(1+e^{-k \rho}\right)$, $q_{(1)}=1-p_{(1)}$;

$$
\begin{aligned}
\tilde{\mu}\left(\bigcup_{j=1}^{n_{k}} \mathscr{U}_{j}\right) & \geq p_{(1)} q_{(1)}+p_{(1)} q_{(1)}^{3}+p_{(1)} q_{(1)}^{5}+\cdots \\
& =p_{(1)} q_{(1)}\left(1+q_{(1)}^{2}+q_{(1)}^{4}+\cdots\right) \\
& =p_{(1)} q_{(1)}\left(\frac{1-q_{(1)}^{2 n_{k}}}{1-q_{(1)}^{2}}\right) \\
& =\frac{q_{(1)}}{1+q_{(1)}}\left(1-\left(\frac{1}{1+e^{-k \rho}}\right)^{2 n_{k}}\right) .
\end{aligned}
$$

[Note: the infinite series gives $q_{(1)} / 1+q_{(1)} \sim \frac{1}{2}$. Therefore we could have defined $n_{k}$ more conveniently by taking it such that the sum of the first $n_{k}$ terms is $\geq \frac{1}{3}$.]

Now $n_{k} \geq e^{k \rho}$, so

$$
\left(\frac{1}{1+e^{-k \rho}}\right)^{2 n_{k}} \leq\left(\frac{1}{1+e^{-k \rho}}\right)^{2 e^{\lambda_{1}}} \sim \frac{1}{e^{2}} \text { for } k \rightarrow \infty .
$$

Thus we can define

$$
\beta=\frac{1}{3}\left(1-\frac{1}{e^{2}}\right)>0 .
$$

Hence Lemma 2.1 applies and the proposition is proved.

REMARK 1. It turns out that Example 1 would have been easier if [3, Theorem 4.4(a)] implied [3, Theorem 4.4(b)]. In particular, this application implies the truth of the above Lemma 2.1, and moreover the condition that the $\left\{\gamma_{j} \mathscr{U}_{j}\right\}$ be disjoint appears unnecessary. Unfortunately, [3, Theorem 4.4(b)] does not follow from [3, Theorem 
4.4(a)]. We give a counterexample, which was motivated by this observation. It was in fact found by first looking for a situation where the $\left\{\gamma_{j} \mathscr{U}_{j}\right\}$ are not disjoint.

We present this counterexample after presenting a method for constructing $\mu$ 's with ratio set contained in $\{0,1, \infty\}$. (This method will also be needed for the counterexample.)

REMARK 2. Here is a special case of Example 1.

Let $e^{\rho}=2$.

Let $\sigma_{i}=k \log 2$ for $i \in\left[2^{k}, 2^{k+1}\right.$ ) (thus $n_{k}=2^{k+1}-2^{k}=2^{k}=e^{\rho k}$ ).

This gives $a_{i}=\left(2^{k}-1\right) /\left(2^{k}+1\right)=1-2 /\left(2^{k}+1\right), i \in\left[2^{k}, 2^{k+1}\right)$.

We have $1 / 2 \in r(X, \Gamma, \mu)$ by the above.

It is easy to verify that any $(d \mu \circ \gamma) / d \mu$ takes on only the values $2^{m}, m \in \mathbb{Z}$ (or possibly $0, \infty$ ).

Thus in fact $\mu$ is type $\mathrm{III}_{\lambda}$, with $\lambda=1 / 2$.

REMARK 3. By choosing rationally independent $\rho_{1}, \rho_{2}$ and including infinitely many block pairs $\left(k \rho_{i}, k \rho_{i},(k+1) \rho_{i}, \ldots(k+1) \rho_{i}\right)$ of both types, we get a $\mu$ of type $\mathrm{III}_{1}$.

We give two examples of product measure of type $\mathrm{II}_{0}$ on an infinite product of two-point spaces.

Example 2 is similar to the type III $_{0}$ example in [11], but we give a short and direct proof that its ratio set is $\{0,1, \infty\}$.

EXAMPLE 2. In the notation of [3], it is clear that everything is determined if the sequence $\left\{\sigma_{i}\right\}$ is specified. Let $\left\{\sigma_{i}\right\}$ be of the form

$$
\ldots, 2^{k}, 2^{k}, \ldots, 2^{k}, 2^{k+1}, 2^{k+1}, \ldots 2^{k+1}, 2^{k+2}, \ldots
$$

where the $n_{k}$ are chosen large enough, to ensure that:

$$
\sum_{i=1}^{\infty}\left(1-a_{i}\right)=\infty .
$$

For example, since $1-a_{i}=2 /\left(1+e^{\sigma_{i}}\right)=2 /\left(1+e^{2^{k}}\right)$ on the $k$ th block, the choice

$$
n_{k} \geq \frac{1}{2}\left(e^{2^{k}}+1\right),\left(n_{k} \in \mathbb{N}\right)
$$

will do. By Moore's criterion [16], this ensures $\mu$ is of type III, for eventually,

$$
\min \left(\frac{2 a_{i}}{1-a_{i}}, 1\right)=1 \quad\left(\text { since } a_{i} \nearrow 1\right) .
$$


So

$$
\sum \min \left(\frac{2 a_{i}}{1-a_{i}}, 1\right)^{2}\left(1-a_{i}\right)=\sum\left(1-a_{i}\right)=\infty .
$$

PROPOSITION 2.2. The ratio set of $\mu$ is contained in $\{0,1, \infty\}$.

PROOF. Suppose not. Let $r \in r(X, \Gamma, \mu), r>2$. Let $\epsilon=1$. Choose $k$ such that $\exp \left(2^{k}\right)>r+1$. Choose $n$ such that $\left\{\sigma_{i}\right\}_{i=n}^{\infty}$ takes values in $\left\{2^{k}, 2^{k+1}, \ldots\right\}$. Let $A=\gamma_{0} X^{n}$ for this $n$ and an! $\gamma_{0}$ ( say $\gamma_{0}=0$ ).

Clearly for any $u \in A$. and $\gamma$ such that $\gamma u \in A$ we get

$$
\frac{d \mu=\gamma}{d \mu}(u)=\exp \sum_{i=n}^{m} \alpha_{i} \sigma_{i}
$$

for some $m$ depending on $y$ and $\alpha,\{-1,0,1\}$. Thus

$$
\sum_{=\infty}^{\infty} \alpha_{i} \sigma_{i} \mid \in\{0\} \cup 2^{k} \mathbb{N}
$$

and so

$$
\frac{d \mu \quad r}{u_{u}}|\omega|-r \mid>1 \quad(\text { since } r>2) \text {. }
$$

Thus there is nower $B$ s revursed hy the definition of $R_{\mu}$.

EXAMPLE 3. ICinunteresumpic to $[3$, Theorem $4.4(a)] \Longrightarrow[3$, Theorem $4.4(\mathrm{~b})]$ and to $[4$, Lemma 221 ,

To define $\left\{\sigma_{1}\right\}$. take hind a in example 2 and insert the value $2^{k}-1$ in front of the $k$ th block for each $k$ Thu $i n$ in a sequence of the form

$$
2^{k-1}, 2^{k-1} \ldots .2 \ldots+21.2^{k}, 2^{k}, \ldots, 2^{k}, 2^{k+1}-1,2^{k+1}, 2^{k+1}, \ldots
$$

The condition on $n_{k}$ is the same as before, for example

$$
n_{k} \geq \exp \left(2^{k}\right) \text {. }
$$

or, more conveniently, define $n_{k}$ instead by considering the series

$$
1+q+q^{2}+\cdots=\frac{1}{1-q}
$$

where $q=\mu_{i}(\{0\})=1 /\left(1+\exp \left(-2^{k}\right)\right)$ (on the block $k$ ) and let $n_{k}$ be the number of terms needed to get a partial sum of say at least $\frac{1}{2}(1-q)^{-1}$. The idea in the form of 
the sequence is that it is essentially like example 2, but with a tiny nick on each big step.

For consistency with the notation of [3] we will use the notation of $G$-measures in the statement below. Recall that for any measure $\mu$ we have

$$
G_{n}=\frac{d \mu}{d \mu^{(n)}} \text { where } \mu^{(n)}=\frac{1}{\left|\Gamma_{n}\right|} \sum_{\gamma \in \Gamma_{n}} \mu \circ \gamma
$$

We then have $g_{n}=G_{n} / G_{n-1}$. In the case of a product measure $\mu=\bigotimes_{n=1}^{\infty} \mu_{n}$, $g_{n}(x)=\mu_{n}\left(\left\{x_{n}\right\}\right)$.

PROPOSITION 2.3. Let $\mu$ be the resulting measure. Then

(i) the ratio set of $\mu$ is contained in $\{0,1, \infty\}$;

(ii) $\forall \epsilon>0, \exists \beta>0$, such that $\forall n \in \mathbb{N}, \forall \gamma_{0} \in \Gamma_{n} \exists L \geq n$ such that: $\mu\left(\left\{u \in \gamma_{0} X^{n}: \exists v \in \gamma_{0} X^{n}\right.\right.$, eventually equal to $u$, such that $l>L$ implies $\left.\left.\left|\prod_{i=n}^{l} g_{i}(v) / g_{i}(u)-e\right|<\epsilon\right\}\right) \geq \beta \mu\left(\gamma_{0} X^{n}\right)$.

REMARK 1. (ii) is the case $r=e$ of [3, Theorem (4.4)(a)]. But by (i), $e$ is not in the ratio set of $\mu$, contradicting [3, Theorem (4.4) (b)].

REMARK 2. It will be evident from the proof that if [3, Theorem (4.4) (a)] is altered to read, instead of $\forall n \exists L$, to ' $\exists L \forall n \ldots l \geq n+L \ldots$ ', then this would not be a counterexample. In fact, the proof given in [3] is valid with this change. Furthermore, this is the version adopted later, in [3, Theorem (5.2)] (where it should read $\left.\sup _{n}(N(n)-n)<K(\epsilon)\right)$.

ProOF (of Proposition 2.3(i)). Suppose not. Let $r>2$ be in the ratio set. Choose $k$ such that $\exp \left(2^{k}\right)>r+1$. Now consider the set $A$ defined as follows. Choose $n \in \mathbb{N}$ such that $\left\{\sigma_{i}\right\}_{i=n}^{\infty}$ is the tail starting from block $k$. Let $i=i$, be the indices where the nicks occur, that is, where

$$
\sigma_{i_{l}}=2^{l}-1, \quad l=1,2, \ldots
$$

Fix a $\gamma_{0} \in \Gamma_{n}$, and define

$$
A=\left\{u \in \gamma_{0} X^{n}: 0=u_{i_{k}}=u_{i_{k+1}}=\cdots\right\}=\left\{u \in \gamma_{0} X^{n}: u_{i_{l}}=0 \quad \forall l \geq k\right\} .
$$

Let us first observe that if $u \in A$ and $\gamma u \in A$ then

$$
\frac{d \mu \circ \gamma}{d \mu}(u)=\exp \left(\sum_{\left.i=n, i \notin \mid i_{i}\right]}^{m} \alpha_{i} \sigma_{i}\right)
$$


because by the definition of $A$, there can be no change in the coordinates $i=i_{l}$, $l=k, k+1, \ldots$ (that is, all those $i$, which are $\geq n$ ).

Here $m \geq n$ and $\alpha_{i} \in\{-1,0,1\}$ depend on $\gamma$ and $u$.

So, since these $\sigma_{i}$ take values in $\left\{2^{k}, 2^{k+1}, \ldots\right\}$ (that is, no $2^{\prime}-1$ type values), we have, as in example 2 ,

$$
\left|\frac{d \mu \circ \gamma}{d \mu}(u)-r\right|>1 \text { for all such } u \text {. }
$$

Hence for this set $A$, and for $\epsilon=1$, there is no set $B$ as required in the definition of $r \in R_{\mu}$.

It remains to check that $\mu(A)>0$. Clearly

$$
\mu(A)=\prod_{i_{i} \geq n} \mu_{i_{i}}(\{0\})=\prod_{i=k}^{\infty} \mu_{i_{i}}(\{0\}) .
$$

But $\mu_{i_{i}}(\{0\})=1 /\left[1+\exp \left(-\sigma_{i_{1}}\right)\right]=1 /\left[1+\exp \left(-\left(2^{l}-1\right)\right)\right]$ since this is the definition of $\sigma_{i}$ on the 'nicks'. This certainly gives a convergent product (since $\sum_{l=k}^{\infty} \exp \left(-2^{l}\right)<$ $\infty$.

Thus $\mu(A)>0$ as required.

Proof of 2.3(ii). Fix $\epsilon>0$. Fix $n \in \mathbb{N}$ and $\gamma_{0} \in \Gamma_{n}$. Consider a $k$ such that the $k$ th block, including its 'nick' occurs inside the tail $\left\{\sigma_{i}\right\}_{i>n}$. We shall do coordinate changes within this block $k$ only, hence we can take the $L$ to be the end of block $k$.

Consider $u \in \gamma_{0} X^{n}$ of the following types:

\begin{tabular}{ccc|ccccccc} 
& & $i_{k}$ & & & & & & $\left(i_{k}+n_{k}\right)$ & \\
$x$ & $x$ & 0 & 1 & $x$ & $x$ & $x$ & $x$ & & $\left(\mathscr{U}_{1}\right)$ \\
$x$ & $x$ & 0 & 0 & 1 & $x$ & $x$ & $x$ & $\vdots$ & $\left(\mathscr{U}_{2}\right)$ \\
$x$ & $x$ & 0 & 0 & 0 & 1 & $x$ & $x$ & $\vdots$ & $\left(\mathscr{U}_{3}\right)$ \\
$x$ & $x$ & 0 & 0 & 0 & 0 & 1 & $x$ & $\vdots$ & $\vdots$ \\
$\vdots$ & $\vdots$ & $\vdots$ & & & & & & $\vdots$ & $\vdots$ \\
$x$ & $x$ & 0 & 0 & $\ldots$ & $\ldots$ & $\ldots$ & $\ldots$ & 01 & $\left(\mathscr{U}_{n_{k}}\right)$
\end{tabular}

For each $\mathscr{U}_{j}$ consider the $\gamma_{j}$ shown below:

\begin{tabular}{ccc|cccccc}
0 & 0 & 1 & 1 & 0 & 0 & 0 & 0 & $\gamma_{1}$ \\
0 & 0 & 1 & 0 & 1 & 0 & 0 & 0 & $\gamma_{2}$ \\
0 & 0 & 1 & 0 & 0 & 1 & 0 & 0 & $\cdots$ \\
0 & 0 & 1 & 0 & 0 & 0 & 1 & 0 & $\gamma_{n_{k}}$
\end{tabular}


Clearly $u \in \mathscr{U}_{j} \rightarrow \gamma_{j} u=: v \in \gamma_{0} X^{n}$ since we have agreed that all our $\mathscr{U}_{j}$ are in $\gamma_{0} X^{n}$.

Let us compute $\left(d \mu \circ \gamma_{j}\right)(u) / d \mu$ for $u \in \mathscr{U}_{j}$. This is just

$$
\frac{\mu_{i_{k}}(\{1\})}{\mu_{i_{k}}(\{0\})} \frac{\mu_{*}(\{0\})}{\mu_{*}(\{1\})}
$$

where * denotes an irrelevant index between $i_{k}$ and $L$ (that is, in the block $k$ ). This coincides with

$$
\prod_{i=n+1}^{L} \frac{g_{i}(v)}{g_{i}(u)}, \text { which reduces to } \frac{e^{-\sigma_{i_{k}}}}{1} \frac{1}{e^{-\sigma_{*}}}=e^{-\left(2^{k}-1\right)} e^{+\left(2^{k}\right)}=e
$$

In other words, it is true, for all $u \in \bigcup_{j=1}^{n_{k}} \mathscr{U}_{j}$, that

$\exists v$ in $\gamma_{0} X^{n}$, eventually equal to $u$, such that $l>L$ implies $\left|\prod_{i=n}^{l} \frac{g_{i}(v)}{g_{i}(u)}-e\right|<\epsilon$

holds. It remains to check that the measure satisfies

$$
\widetilde{\mu}\left(\bigcup_{j=1}^{n_{k}} \mathscr{U}_{j}\right) \geq \beta \quad \text { for some absolute } \beta>0 .
$$

We have

$$
\begin{aligned}
& \tilde{\mu}\left(\mathscr{U}_{1}\right)=\mu_{i_{k}}(\{0\}) \mu_{*}(\{1\}) \\
& \tilde{\mu}\left(\mathscr{U}_{2}\right)=\mu_{i_{k}}(\{0\}) \mu_{*}(\{0\}) \mu_{*}(\{1\}) \\
& \tilde{\mu}\left(\mathscr{U}_{3}\right)=\mu_{i_{k}}(\{0\}) \mu_{*}(\{0\}) \mu_{*}(\{0\}) \mu_{*}(\{1\})
\end{aligned}
$$

and the $\mathscr{U}_{j}$ are all disjoint. Thus, we get a total of

$$
q_{i_{k}} p_{(k)}\left(1+q_{(k)}+q_{(k)}^{2}+\cdots\right)
$$

where $q_{i_{k}}=\mu_{i_{k}}(\{0\}), p_{(k)}=\mu_{*}(\{1\}), q_{(k)}=1-p_{(k)}$.

We agreed that $n_{k}$ was large enough to give us at least

$$
q_{i_{k}} p_{(k)} \frac{1}{2} \frac{1}{1-q_{(k)}}=q_{i_{k}} \frac{1}{2} \geq \frac{1}{3}
$$

since $q_{i_{k}}=1 /\left[1+\exp \left(-\left(2^{k}-1\right)\right)\right]$ is nearly equal to 1 .

Hence we can take $\beta=\frac{1}{3}$. This proves 2.3 (ii). 
REMARK. Notice how this fits in with Lemma 2.1. There it was required also that $\left\{\gamma_{j} \mathscr{U}_{j}\right\}$ be disjoint. Here we can check directly how badly they fail to be disjoint (of course, they must fail, because otherwise Lemma 2.1 would be contradicted).

The $\gamma_{j} \mathscr{U}_{j}$ are:

$\begin{array}{cccccc}1 & 0 & x & x & x & \gamma_{1} \mathscr{U}_{1} \\ 1 & 0 & 0 & x & x & \gamma_{2} \mathscr{U}_{2} \\ 1 & 0 & 0 & 0 & x & \vdots \\ 1 & 0 & 0 & 0 & 0 & \vdots\end{array}$

and so on. Clearly all are subsets of the first one, which has $\tilde{\mu}\left(\gamma_{1} \mathscr{U}_{1}\right)$ very small $\left(\leq \mu_{i_{k}}(\{1\})\right)$.

\section{Proof of a proposition}

The following proposition was given as [3, Proposition 6.2]. Unfortunately, its proof used [3, Theorem 4.4], which we have just disproved! Here is a corrected proof, not without interest in its own right.

PROPOSITION 3.1. If $a_{i} \rightarrow 0$ and $\sum a_{i}^{2}=\infty$, then the measure $\mu$ is of type $\mathrm{III}_{1}$, that is, its ratio set is $[0, \infty]$.

We need the following probabilistic lemma. First let us fix some notation as in [3, pp. 11-12]. For given $0 \leq a_{i} \leq 1 / 2, i=1,2, \ldots$ we associate the sequence $\sigma_{i}=\log \left\{\left(1+a_{i}\right) /\left(1-a_{i}\right)\right\} \quad\left(a_{i} \leq \sigma_{i} \leq 4 a_{i}\right)$ and vice versa (that is, if a statement refers to $\left\{\sigma_{i}\right\}$ first, we assume $\left\{a_{i}\right\}$ defined in terms of $\left.\left\{\sigma_{i}\right\}\right)$.

We also associate with a given $\left\{a_{i}\right\}$ independent random variables as follows. These are $\left\{u_{i}\right\}$ and $\left\{v_{i}\right\}$, where

$$
P\left(u_{i}=0\right)=P\left(v_{i}=0\right)=\frac{1+a_{i}}{2}, \quad P\left(u_{i}=1\right)=P\left(v_{i}=1\right)=\frac{1-a_{i}}{2},
$$

and all are taken to be independent (thus $\left\{u_{i}\right\}$ are independent and $\left\{v_{i}\right\}$ is an independent copy of the sequence $\left.\left\{u_{i}\right\}\right)$. Here as usual $P(*)$ is the probability of the event $*$.

Now put $\Delta_{i}=v_{i}-u_{i}$. Then $\Delta_{i} \in\{-1,0,1\}$ and $E\left(\Delta_{i}\right)=0$. A simple calculation shows that $E\left(\Delta_{i}^{2}\right)=\frac{1}{2}\left(1-a_{i}^{2}\right)$.

Also define for $1 \leq n \leq m$, (and for given $\left\{a_{i}\right\}$ ),

$$
S_{n}^{m}=\sum_{i=n}^{m} \sigma_{i} \Delta_{i}=\sum_{i=n}^{m} \sigma_{i}\left(v_{i}-u_{i}\right)
$$


LEMMA 3.1. Given $-\infty<a<b<\infty$, there exist $p:=p(a, b)>0$ and $1 \geq \delta:=\delta(a, b)>0$ such that given any $\left\{a_{i}\right\}$ and any $m \in \mathbb{N}$ satisfying: $0 \leq \sigma_{i} \leq$ $\delta, 1 \leq i \leq m$, and $2 \leq \sum_{i=1}^{m} \sigma_{i}^{2} \leq 2+\delta^{2}$, it follows that:

$$
P\left(a \leq S_{1}^{m} \leq b\right) \geq p .
$$

REMARKS. (1) We emphasize that $p$ and $\delta$ depend only on the given interval $[a, b]$. So the conclusion holds 'uniformly', whenever $\left\{a_{i}\right\}_{i=1}^{m}$ has the stated properties. (2) Obviously, if instead of ' $1 \leq i \leq m$ ' we consider ' $n \leq i \leq m$ ', we get the same result (applying the lemma): If $0 \leq \sigma_{i} \leq \delta, n \leq i \leq m$, and $2 \leq \sum_{i=n}^{m} \sigma_{i}^{2} \leq 2+\delta^{2}$, then

$$
P\left(a \leq S_{n}^{m} \leq b\right) \geq p,
$$

since the index $i$ plays no role in the statement. (Here $\delta=\delta(a, b), p=p(a, b)$ ).

(3) The $\left\{\Delta_{i}\right\}$ are independent, but not identically distributed; the distribution of $\Delta_{i}$ is given by $a_{i}$. However, the distributions are all 'comparable' since $a_{i} \leq 1 / 2$ (in fact $a_{i} \leq \sigma_{i} \leq \delta$ imposes an even stronger uniformity on them if $\delta$ is small.)

(4) If $\delta$ is small, then $m$ must be large (at least $1 / \delta^{2}$ ). Thus $S_{1}^{m}$ is an essentially normalized sum of a large number of independent random variables.

PROOF OF LEMMA. The lemma follows from an exercise in [6, problem 5, page 205]. This exercise asserts the following: For every $\epsilon>0$ there is a $\delta=\delta(\epsilon)>0$ such that whenever $k \in \mathbb{N}$ and $X_{1}, \ldots, X_{k}$ are independent zero mean random variables with

$$
\sum_{i=1}^{-k} E\left(X_{i}^{2}\right)=1, \sum_{i=1}^{k} E\left(\left|X_{i}\right|^{3}\right) \leq \delta, \quad \text { and } S:=\sum_{i=1}^{k} X_{i},
$$

then $\sup _{x \in \mathbb{R}}|P(S \leq x)-\Phi(x)| \leq \epsilon$ where $\Phi(x)=\int_{-\infty}^{x} e^{-r^{2} / 2} d t / \sqrt{2 \pi}$ is the standard normal distribution function.

This is simply a quantitative version of Liapunov's Central Limit Theorem and can be verified by following the steps in the proof of it given in [6, Theorem 7.1.2]. (A more direct proof can be given for our random variables $\Delta_{i}$ but we omit it).

Given the above result, the lemma follows by normalizing $S_{1}^{m}$ and substituting it for the $S$ in the above result from [6]: (indeed $S_{1}^{m}$ is almost normalized, but not quite, because of the $\delta$ ).

Given $-\infty<a<b<\infty$, choose $a<a^{\prime}<b^{\prime}<b$ (say $a^{\prime}=\frac{3}{4} a+\frac{1}{4} b$, $b^{\prime}=\frac{1}{4} a+\frac{3}{4} b$ to be definite). Let $p^{\prime}=\Phi\left(b^{\prime}\right)-\Phi\left(a^{\prime}\right)=\int_{a^{\prime}}^{b^{\prime}} e^{-t^{2} / 2} d t / \sqrt{2 \pi}$ and put $\epsilon^{\prime}=p^{\prime} / 100, \delta^{\prime}=\delta\left(\epsilon^{\prime}\right)$.

To obtain the lemma, choose $\delta_{0}<1$ and small enough to ensure

$$
a \leq a^{\prime} \sqrt{1 \pm \delta_{0}^{2}}<b^{\prime} \sqrt{1 \pm \delta_{0}^{2}} \leq b
$$


and put

$$
\delta(a, b)=\frac{1}{1000} \min \left(\delta_{0}, \delta^{\prime}\right)
$$

We shall compute the resulting $p=p(a, b)$. Assume $\sigma_{i} \leq \delta(a, b):=: \delta$ and $2 \leq \sum_{i=1}^{m} \sigma_{i}^{2} \leq 2+\delta^{2}$ as in the statement of the lemma.

To normalize $S_{1}^{m}$, we need the variance

$$
E\left(\left(S_{1}^{m}\right)^{2}\right)=\sum_{i=1}^{m} E\left(\sigma_{i}^{2} \Delta_{i}^{2}\right)=\sum_{i=1}^{m} \sigma_{i}^{2}\left(\frac{1-a_{i}^{2}}{2}\right)
$$

Since $\sigma_{i} \leq \delta(a, b)=: \delta$, and $2 \leq \sum_{i=1}^{m} \sigma_{i}^{2} \leq 2+\delta^{2}$, we have $a_{i}^{2} \leq \sigma_{i}^{2} \leq \delta(a, b)^{2}$.

Thus

$$
\frac{1}{2} \sum_{i=1}^{m} \sigma_{i}^{2} \geq \sum_{i=1}^{m} \sigma_{i}^{2}\left(\frac{1-a_{i}^{2}}{2}\right) \geq \frac{1}{2} \sum_{i=1}^{m} \sigma_{i}^{2}-\frac{1}{2} \delta^{2} \sum_{i=1}^{m} \sigma_{i}^{2} \geq 1-\delta^{2},
$$

that is,

$$
1-\delta^{2} \leq E\left(\left(S_{1}^{m}\right)^{2}\right) \leq 1+\frac{1}{2} \delta^{2} .
$$

Putting $X_{i}=\sigma_{i} \Delta_{i} / \sqrt{E\left(\left(S_{1}^{m}\right)^{2}\right)}$ and $S=\sum_{i=1}^{m} X_{i}$, we have that

$$
\left|X_{i}\right|^{3}=\left\|X_{i}\right\|_{\infty} \cdot\left|X_{i}\right|^{2} \leq \max _{i}\left(\sigma_{i}\right) \cdot \frac{1}{\sqrt{1-\delta^{2}}}\left|X_{i}\right|^{2} \leq \frac{\delta}{\sqrt{1-\delta^{2}}} X_{i}^{2} .
$$

Thus

$$
\sum_{i=1}^{m} E\left(\left|X_{i}\right|^{3}\right) \leq \frac{\delta}{\sqrt{1-\delta^{2}}} \leq 2 \delta \leq \delta^{\prime}=\delta\left(\epsilon^{\prime}\right) .
$$

Hence Chung's exercise applies with $\epsilon=\epsilon^{\prime}$. Thus

$$
\begin{aligned}
P\left(a^{\prime}<S<b^{\prime}\right) & =P\left(S<b^{\prime}\right)-P\left(S \leq a^{\prime}\right) \\
& \geq\left(\Phi\left(b^{\prime}\right)-\Phi\left(a^{\prime}\right)\right)-2 \epsilon^{\prime} \\
& =p^{\prime}-2 p^{\prime} / 100=0.98 p^{\prime}
\end{aligned}
$$

If $S<b^{\prime}$, then $S_{1}^{m}=S \sqrt{\left.E\left(S_{1}^{m}\right)^{2}\right)}<b^{\prime} \sqrt{1+d^{2} / 2} \leq b^{\prime} \sqrt{1+\delta_{0}^{2}} \leq b$. If $a^{\prime}<S$, then $a^{\prime} \sqrt{1-\delta^{2}}<S_{1}^{m}$, so $a \leq S_{1}^{m}$. It follows that if $a^{\prime}<S<b^{\prime}$ then $a \leq S_{1}^{m} \leq b$, and we conclude that

$$
P\left(a \leq S_{1}^{m} \leq b\right) \geq P\left(a^{\prime}<S<b^{\prime}\right) \geq 0.98 p^{\prime}
$$


Thus we can take

$$
p(a, b)=0.98 p^{\prime}=0.98 \int_{\frac{3}{4} a+\frac{1}{4} b}^{\frac{1}{4} a+\frac{1}{4} b} e^{-t^{2} / 2} d t / \sqrt{2 \pi}
$$

and the lemma is proved.

PROOF OF PROPOSITION 3.1. It suffices to show the following, which is a version of [3, Proposition 2.3 (i)]:

For any $0<r<\infty, \epsilon>0$, put $\rho=\log r$. Then for every $A \subset X$ with $\mu(A)>0$, there exists $\gamma \in \Gamma$ such that

$$
\mu\left(\left\{x \in A: \gamma x \in A \text { and }\left|\log \frac{d \mu \circ \gamma}{d \mu}(x)-\rho\right| \leq \epsilon\right\}\right)>0 .
$$

Recall that if $u=\left(u_{i}\right)_{i=1}^{\infty}, \gamma u=\left((\gamma u)_{i}\right)_{i=1}^{\infty}:=:\left(v_{i}\right)_{i=1}^{\infty}$ then

$$
\frac{d \mu \circ \gamma}{d \mu}(u)=\prod_{i=1}^{\infty} \frac{g_{i}\left((\gamma u)_{i}\right)}{g_{i}\left(u_{i}\right)}=\prod\left(\frac{1+a_{i}}{1-a_{i}}\right)^{u_{i}-(\gamma u)_{i}},
$$

W) that

$$
\log \frac{d \mu \circ \gamma}{d \mu}(u)=-\sum_{i=1}^{\infty} \sigma_{i}\left((\gamma u)_{i}-u_{i}\right)
$$

Thus, consider the interval, (for given $\rho, \epsilon$ ), $[-\rho-\epsilon,-\rho+\epsilon]=:[a, b]$.

Let the set $A \subset X$ be given, $\mu(A)>0$. Let $\kappa=p(a, b) / 200$ where $p(a, b)$ is Jetined in Lemma 3.1. Let $n_{1} \in \mathbb{N}$ be large enough to ensure that

$$
\text { if } n \geq n_{1} \text { then } \sigma_{n} \leq \delta(a, b)
$$

where $\delta(a, b)$ is also defined in Lemma 3.1.

Since $\mu(A)>0$, we can find $n \geq n_{1}$ and $\gamma_{0} \in \Gamma_{n}$ such that the cylinder $\gamma_{0} X^{n}$ satisfies

$$
\mu\left(A \cap \gamma_{0} X^{n}\right) \geq(1-\kappa) \mu\left(\gamma_{0} X^{n}\right) .
$$

Put $\tilde{\mu}=\mu / \mu\left(\gamma_{0} X^{n}\right)$ restricted to $\gamma_{0} X^{n}, A_{0}=A \cap \gamma_{0} X^{n}, X_{0}=\gamma_{0} X^{n}$.

Consider $X_{0} \times X_{0}$ with measure $\tilde{\mu} \times \tilde{\mu}$. Observe that

$$
A_{0} \times A_{0} \subset X_{0} \times X_{0} \text { and }(\tilde{\mu} \times \tilde{\mu})\left(A_{0} \times A_{0}\right) \geq(1-\kappa)^{2} \geq 1-2 \kappa .
$$

Let $(u, v) \in X_{0} \times X_{0}$, and identify

$$
u=\left(u_{n}, u_{n+1}, \ldots\right) \text { and } v=\left(v_{n}, v_{n+1}, \ldots\right) .
$$


Then $\left\{u_{i}\right\}_{i=n}^{\infty},\left\{v_{i}\right\}_{i=n}^{\infty}$ are independent random variables on the probability space $\left(X_{0} \times\right.$ $X_{0}, \tilde{\mu} \times \tilde{\mu}$ ) satisfying all the conditions of the Lemma (in the same notation). Since

$$
\sigma_{i} \leq \delta(a, b), \quad i \geq n,
$$

we can also choose $m>n$ large enough (but not too large) so that

$$
2 \leq \sum_{i=1}^{m} \sigma_{i}^{2} \leq 2+\delta(a, b)^{2} .
$$

Since $\sum_{i=n}^{\infty} \sigma_{i}^{2}=\infty$, this is achieved by letting $m$ be the smallest integer such that

$$
2 \leq \sum_{i=n}^{m} \sigma_{i}^{2}
$$

Thus, applying the Lemma to

$$
S_{n}^{m}=\sum_{i=n}^{m} \sigma_{i}\left(v_{i}-u_{i}\right)
$$

we have

$$
(\tilde{\mu} \times \tilde{\mu})\left(a \leq S_{n}^{m} \leq b\right) \geq p(a, b) .
$$

Let $G_{n}^{m}$ denote the 'good set'

$$
G_{n}^{m}=\left\{(u, v) \in X_{0} \times X_{0}: a \leq S_{n}^{m}(u, v) \leq b\right\}
$$

so that

$$
\tilde{\mu} \times \tilde{\mu}\left(G_{n}^{m}\right) \geq p(a, b) .
$$

Since $S_{n}^{m}$ depends only on the coordinates $n, \ldots, m$, then $G_{n}^{m}$ is a disjoint union of cylinder sets

$$
G_{n}^{m}=\bigcup_{\alpha}\left(U_{\alpha} \times V_{\alpha}\right)
$$

where each $U_{\alpha}, V_{\alpha}$ is of the form

$$
\begin{aligned}
& U_{\alpha}=\left(u_{n}^{\alpha}, u_{n+1}^{\alpha}, \ldots, u_{m}^{\alpha}\right) \times\{0,1\} \times\{0,1\} \times \cdots \\
& V_{\alpha}=\left(v_{n}^{\alpha}, v_{n+1}^{\alpha}, \ldots, v_{m}^{\alpha}\right) \times\{0,1\} \times\{0,1\} \times \cdots
\end{aligned}
$$

(where the $u_{i}^{\alpha}, v_{i}^{\alpha} i=n, \ldots, m$ are the 'good' choices of zeros and ones). 
Our problem is to show that there is an $\alpha=\alpha_{0}$ such that the $\gamma=\gamma_{\alpha_{0}}$, determined by sending $u_{i}^{\alpha_{0}} \mapsto v_{i}^{\alpha_{0}}, i=n, \ldots, m$, satisfies property (3.1).

Put $P=\tilde{\mu} \times \tilde{\mu}$ for convenience. We have

$$
\begin{aligned}
P\left(G_{n}^{m} \cap\left(A_{0} \times A_{0}\right)\right) & =P\left(G_{n}^{m}\right)-P\left(G_{n}^{m} \backslash\left(A_{0} \times A_{0}\right)\right) \\
& \geq P\left(G_{n}^{m}\right)-P\left(\left(X_{0} \times X_{0}\right) \backslash\left(A_{0} \times A_{0}\right)\right) \\
& \geq P\left(G_{n}^{m}\right)-2 \kappa=P\left(G_{n}^{m}\right)-0.01 p(a, b) \\
& \geq P\left(G_{n}^{m}\right)-0.01 P\left(G_{n}^{m}\right) \\
& =0.99 P\left(G_{n}^{m}\right),
\end{aligned}
$$

that is, $A_{0} \times A_{0}$ covers $99 \%$ or more of the good set, $G_{n}^{m}$.

Consequently, $A_{0} \times A_{0}$ covers $99 \%$ or more of at least one of the $U_{\alpha} \times V_{\alpha}$ (whose disjoint union is $G_{n}^{m}$ ).

Thus, there exists $\alpha_{0}$ such that

$$
P\left(\left(A_{0} \times A_{0}\right) \cap\left(U_{\alpha_{0}} \times V_{\alpha_{0}}\right)\right) \geq 0.99 P\left(U_{\alpha_{0}} \times V_{\alpha_{0}}\right)
$$

where $U_{\alpha_{0}} \times V_{\alpha_{0}} \subset G_{n}^{m}$.

Put $U_{0}=U_{\alpha_{0}}, V_{0}=V_{\alpha_{0}}$. We have

$$
\left.\frac{\mu\left(A_{0} \cap U_{0}\right) \mu\left(A_{0} \cap V_{0}\right)}{\mu\left(U_{0}\right) \mu\left(V_{0}\right)}=\frac{P\left(\left(A_{0} \cap U_{0}\right) \times\left(A_{0} \cap V_{0}\right)\right)}{P\left(U_{0} \times V_{0}\right)} \geq 0.99 \quad \text { (since } P=\tilde{\mu} \times \tilde{\mu}\right) .
$$

It follows that

$$
\frac{\mu\left(A_{0} \cap U_{0}\right)}{\mu\left(U_{0}\right)} \geq 0.99 \text { and } \frac{\mu\left(A_{0} \cap V_{0}\right)}{\mu\left(V_{0}\right)} \geq 0.99 .
$$

Define the proposed $\gamma \in \Gamma$ by the conditions

$$
\begin{aligned}
& (\gamma x)_{i}=x_{i} \quad \text { if } \quad i<n \quad \text { or } \quad i>m \text {, } \\
& (\gamma x)_{i}=v_{i}^{\alpha_{11}} \quad \text { if } \quad x_{i}=u_{i}^{\alpha_{0}} \quad \text { and } \quad n \leq i \leq m .
\end{aligned}
$$

Clearly $\gamma\left(U_{0}\right)=V_{0}$ and

$$
\frac{\mu(E)}{\mu\left(U_{0}\right)}=\frac{\mu(\gamma(E))}{\mu\left(V_{0}\right)} \quad \text { whenever } E \subset U_{0} .
$$

Thus

$$
\frac{\mu\left(\gamma\left(A_{0} \cap U_{0}\right)\right)}{\mu\left(V_{0}\right)}=\frac{\mu\left(A_{0} \cap U_{0}\right)}{\mu\left(U_{0}\right)} \geq 0.99
$$

Combining this knowledge with the above fact that $\mu\left(A_{0} \cap V_{0}\right) / \mu\left(V_{0}\right) \geq 0.99$, we get

$$
\frac{\mu\left(\gamma\left(A_{0} \cap U_{0}\right) \cap\left(A_{0} \cap V_{0}\right)\right)}{\mu\left(V_{0}\right)} \geq 0.99-0.01=0.98>0 .
$$


Put $B=\gamma^{-1}\left(\gamma\left(A_{0} \cap V_{0}\right) \cap\left(A_{0} \cap V_{0}\right)\right)$. Then

$$
\mu(B)>0, B \subset A_{0} \cap U_{0} \subset A_{0} \subset A, \gamma(B) \subset A_{0} \cap V_{0} \subset A_{0} \subset A,
$$

and $B \times \gamma(B) \subset U_{0} \times V_{0} \subset G_{n}^{m}$, that is, if $u \in B$, then

$$
-\sum_{i=n}^{m}\left((\gamma u)_{i}-u_{i}\right) \sigma_{i} \in[\log r-\epsilon, \log r+\epsilon]
$$

which is the required property (3.1)

\section{Markov measures of type $\mathrm{III}_{0}$}

We now present a class of examples which are measured odometers, but with measures which are not product measures, but rather Markov measures.

We use the Daniell-Kolmogorov consistency theorem to define a measure on $X=$ $\{0,1\}^{\mathbb{Z}^{+}}$by specifying the measure of each cylinder. To be specific, let $\left[x_{0} x_{1} \ldots x_{n}\right]$ denote the set of points in $X$ whose first $n+1$ coordinates are $x_{0}, x_{1}, \ldots, x_{n}$. A set of this kind will be called an $n$-cylinder and will also be denoted $[x]^{n}$. Then define

$$
\mu\left(\left[x_{0} x_{1} \ldots x_{n}\right]\right)=\frac{1}{2} P_{x_{0} x_{1}}^{(1)} P_{x_{1} x_{2}}^{(2)} \ldots P_{x_{n-1} x_{n}}^{(n)}, \quad \text { where } P_{i j}^{(k)}= \begin{cases}1-q_{k} & \text { if } i=j \\ q_{k} & \text { if } i \neq j .\end{cases}
$$

This gives a measure on $X$. We then define the transformation group on the space: $\Gamma$ is the group of all finite coordinate rotations of $X$ generated by the $\gamma_{n}$ as introduced above.

We will now demonstrate briefly an orbit equivalent system which in some ways resembles the more familiar systems. Define a second measure $v$ on $X$ which is just a product measure:

$$
v\left(\left[x_{0} x_{1} \ldots x_{n}\right]\right)=\frac{1}{2} q_{x_{l}}^{(1)} \ldots q_{x_{n}}^{(n)}, \quad \text { where } q_{i}^{(k)}= \begin{cases}1-q_{k} & \text { if } i=0 \\ q_{k} & \text { if } i=1 .\end{cases}
$$

We then define a second transformation group on the space: $\Gamma^{\prime}$ is the group of all finite coordinate changes on $X$ which change an even number of coordinates (this is generated by the $\left.\gamma_{m} \circ \gamma_{n}\right)$. Then we can show that the $\Gamma$ action on $(X, \mu)$ is orbit equivalent to the $\Gamma^{\prime}$ action on $(X, v)$. The equivalence is given by a map which is in fact a measure-preserving homeomorphism. Namely, define $\Phi: X \rightarrow X$ by $\Phi(x)_{n}=x_{0}+x_{1}+\cdots+x_{n} \bmod 2$. If $x_{0}, \ldots, x_{n}$ are given, define $y_{0}, \ldots, y_{n}$ by $y_{k}=x_{0}+x_{1}+\cdots+x_{k}(\bmod 2)$. Then a quick check shows that $P_{y_{k-1} y_{k}}^{(k)}=q_{x_{k}}^{(k)}$ so it 
follows that $v\left(\left[x_{0} \ldots x_{n}\right]\right)=\mu\left(\left[y_{0} \ldots y_{n}\right]\right)$. This shows that $\Phi$ is measure-preserving as required. We can write down an explicit inverse $\Psi$ as follows: $\Psi(y)_{n}=y_{n-1}+y_{n}$ mod 2, thereby showing that $\Phi$ is a homeomorphism (and in particular is invertible). To check that $\Phi$ is an orbit equivalence, from $\left(X, \nu, \Gamma^{\prime}\right)$ to $(X, \mu, \Gamma)$, pick an $x \in X$ and note that $\Phi\left(\gamma_{m} \circ \gamma_{n}(x)\right)$ differs from $\Phi(x)$ in all coordinates between the $m$ th and the $n$-1st. So the image of a $\Gamma$ orbit under $\Phi$ is exactly a $\Gamma$ orbit as required.

Note that the system $(X, \nu, \Gamma)$ is known to have an associated flow with the AT property by Hawkins' result. (In fact, more is true: the flow on $X \times \mathbb{R}$ prior to forming the quotient also has the AT property.) But the system which we are considering, $(X, \mu, \Gamma)$, is not orbit equivalent to the above, but rather to $\left(X, \nu, \Gamma^{\prime}\right)$ which appears at first sight to be very similar to $(X, v, \Gamma)\left(\Gamma^{\prime}\right.$ is a subgroup of $\Gamma$ of index 2$)$, but Hawkins' proof does not seem to work in this situation where there is more dependence.

The system as defined so far has a number of parameters $q_{n}$. We now show how to choose them in such a way that the system is a $\mathrm{III}_{0}$ system by analogy with the construction in Section 2. We will construct an increasing sequence of integers $n_{i}$ and a rapidly decreasing sequence of real numbers $p_{i}$ and define

$$
q_{n}= \begin{cases}\frac{1}{2} & \text { if } n=0 \\ p_{i} & \text { if } n_{i-1}<n \leq n_{i} .\end{cases}
$$

Defining $n_{0}=0$ and $m_{i}=n_{i}-n_{i-1}$, the sequences are chosen so that

$$
\begin{gathered}
\sum_{i=1}^{\infty} m_{i} p_{i}=\infty \\
R_{j}>1 \text { and } \lim _{j \rightarrow \infty} R_{j}=\infty \quad \text { where } R_{j}=\prod_{i<j}\left(\frac{p_{i}}{1-p_{i}}\right)^{m_{i}} \cdot \frac{1-p_{i}}{p_{j}} .
\end{gathered}
$$

We remark that it is possible to simultaneously satisfy these conditions by an inductive construction. Supposing $n_{1}, \ldots, n_{k}$ and $p_{1}, \ldots, p_{k}$ are chosen. Then $p_{k+1}$ may be chosen to ensure that $R_{k+1}>k+1$ and subsequently, $m_{k+1}$ may be chosen so that $m_{k+1} p_{k+1}>1$.

The first condition, (4.1) is to ensure that the system is not of type I. The condition is that the expected number of transitions (that is, places at which $x_{i} \neq x_{i-1}$ ) is infinite. By the Kolmogorov 0-1 law, this ensures that the probability of having a sequence which is eventually all $1 \mathrm{~s}$ or eventually all $0 \mathrm{~s}$ is 0 and this is sufficient to guarantee that the system is not of type $\mathrm{I}$.

The second condition, (4.2) is to ensure that the only ratios occurring in the ratio set are 0,1 and $\infty$. Further, it can be checked as in Section 2, that all these occur so that the system really is of type III $_{0}$. Given $\gamma \in \Gamma$ and $x \in X$, we see that the ratio $d \mu \circ \gamma / d \mu$ is constant on any $n_{k}$-cylinder about $x$ where $\gamma$ only affects coordinates before the $n_{k}$ th and so $d \mu \circ \gamma / d \mu(x)=\mu\left([\gamma(x)]^{n_{k}}\right) / \mu\left([x]^{n_{k}}\right)$. 
Given $x \in X$, define its block type as follows: the block type is a sequence of numbers $\left(a_{0}, a_{1}, a_{2}, \ldots\right)$ where $a_{0}=x_{0}$ and $a_{i}$ denotes the number of transitions in block $i$ (that is the number of $n$ with $n_{i-1}<n \leq n_{i}$ such that $a_{n} \neq a_{n-1}$, where $n_{0}$ is taken to be 0 ). The number $a_{0}$ is to be interpreted as the number of transitions in the Oth block (that is, we have a notional initial state of 0 and then $a_{0}$ denotes the number of transitions from 0 in the $0 \mathrm{~h}$ block.) Next, we note that the measure of a cylinder set of the form $[x]^{n_{t}}$ is determined by its block type. If the block type is $\left(a_{0}, a_{1}, a_{2} \ldots\right)$ then the measure of $\left[\left.x\right|^{n} \cdot\right.$ is $\prod_{i=1}^{4} p_{i}^{a_{i}}\left(1-p_{i}\right)^{m_{i}-a_{i}}$. If $\gamma$ is as above and $\gamma(x)$ has block type $\left(b_{0}, b_{1}, \ldots, b_{4}, a_{1}, \ldots a_{4}, \ldots \ldots\right)$ then we see that

$$
\left.\frac{d \mu \circ \gamma}{d \mu}(x)=\mu(1: 1 x) \gamma^{\prime}\right) / \mu\left([x]^{n_{k}}\right)=\left(\frac{p_{1}}{1-p_{1}}\right)^{b_{1}-a_{1}} \cdots\left(\frac{p_{k}}{1-p_{k}}\right)^{b_{k}-a_{k}} .
$$

We then see that either all of the terms $b_{i}-a_{i}$ are 0 (in which case the ratio is 1 ) or there is a largest i for which $b-a_{i}$ is non-zero. It is then straightforward to check that the ratio is either larger thun $R_{\mathrm{k}}$ or smaller than $1 / R_{k}$ according to whether $b_{i}-a_{i}$ is negative or posituc Simc in the definition of a ratio set, the ratios are required to be found in an set $f$ " 1 mmituse measure, letting $A$ be an $n_{k}$-cylinder, if $B$ is a subset of $A$ and $\gamma(B) \subset A$ then the rallo $d \mu \circ \gamma / d \mu$ on $B$ is either 1 or larger than $R_{k}$ or smaller than $1, R$. Cimsercly. inside any set of positive measure, there are ratios which are arbitranl? , har 1,1 , and $x$. This proves that the system is of class $\mathrm{III}_{0}$.

We use a constru twon wh Hamachi and Osikawa [9] to give an explicit description of the associated flim . : the …em $(X, v, \Gamma)$ and show that it still has the AT property. To construct the flins it . inst neciessary to get an explicit description of a quotient

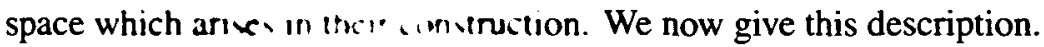

Since the funithm $\therefore, d d \mu$ are continuous, they are defined on the whole space and not just on $x$. . nkessure 1 . This means that the following definition makes sense. Given : and , in $x$. write $x \sim y$ if $y=\gamma(x)$ for some $\gamma \in \Gamma$ and $d \mu \circ \gamma / d \mu(x)=1$. Then let ) denote the collection of equivalence classes $X / \sim$ and let $\Pi$ denote the natural projection from $X$ to $Y$. The $\sigma$-algebra on $Y$ is then given by $\mathscr{F}=\left\{A \subset Y: \Pi^{-1} A \in \mathscr{H} \mid\right.$. Let $\mathscr{G}$ denote the collection of measurable subsets of $X$ which are unions of $\sim$-equivalence classes. Then we see that $\mathscr{F}=\Pi(\mathscr{G})$.

We are then able to identify certain elements of $\mathscr{G}$. We first note that if $x$ has block type $\left(a_{0}, a_{1}, a_{2}, \ldots\right)$ and $y$ has block type $\left(b_{0}, b_{1}, b_{2}, \ldots\right)$ then

$$
\frac{\mu\left([y]^{n_{1}}\right)}{\mu\left([x]^{n_{4}}\right)}=\left(\frac{p_{1}}{1-p_{1}}\right)^{b_{1}-a_{1}} \cdots\left(\frac{p_{k}}{1-p_{k}}\right)^{b_{k}-a_{k}} .
$$

In particular, by the remarks made in the section on the $\mathrm{III}_{0}$ property, we see that $x \sim y$ if and only if $x$ and $y$ lie in the same orbit and have $a_{i}=b_{i}$ for all $i \geq 1$. We now show that if $x \sim y$ then $a_{0}=b_{0}$ as well. To see this, suppose $x \sim y$. Then as 
noted above, $a_{i}=b_{i}$ for each $i \geq 1$. This means that $x_{n_{i}}=x_{n_{i-1}}+a_{i}(\bmod 2)$ and $y_{n_{i}}=y_{n_{i-1}}+a_{i}(\bmod 2)$ for each $i \geq 1$. In particular it follows that $y_{n_{i}}-x_{n_{i}} \bmod 2$ is independent of $i$ for $i \geq 0$. Since $x$ and $y$ live in the same orbit, we require that $x$ and $y$ differ only in finitely many places, so in particular, $x_{n_{i}}=y_{n_{i}}$ for all $i \geq 0$. This implies that $a_{0}=b_{0}$. Denote the block type of $x$ by $B(x)$. We have shown that $x \sim y \Longrightarrow B(x)=B(y)$. This allows us to identify certain elements of $\mathscr{G}$ as follows. Set $Z=\left\{\left(a_{0}, a_{1}, \ldots\right): 0 \leq a_{0} \leq 1 ; 0 \leq a_{i} \leq m_{i}\right\}$. Given $b \in Z$, write $C_{k}(b)$ for $\left\{x: B\left(x_{i}\right)=b_{i}, \forall i \leq k\right\}$. Then $C_{k}(b)$ is a finite union of cylinder sets of length $n_{k}$ so is certainly a measurable set. If $x \in C_{k}(b)$ and $x \sim y$, then since $y$ has the same block type as $x$, we see $y \in C_{k}(b)$ so $C_{k}(b)$ is a union of $\sim$-equivalence classes as required. It will be useful to note that by the above arguments, if $x$ and $y$ are members of $C_{k}(b)$ then $x_{n_{i}}=y_{n_{i}}$ for each $i \leq k$.

We have therefore identified a collection of cylinder type sets which belong to $\mathscr{G}$. It is then possible to show that these sets generate $\mathscr{G}$. We demonstrate this by showing that the algebra consisting of finite unions of sets of the form $C_{k}(b)$ may be used to approximate any element of $G$.

Let $A$ be any element of $\mathscr{G}$. We will show that $A$ may be arbitrarily closely approximated by taking a union of sets of the form $C_{k}(b)$. Let $\epsilon>0$ be given. Then pick $\delta<\min (1, \epsilon / 2)$. Then let $\mathscr{A}$ denote the algebra of all finite unions of cylinder sets in $X$. Then since $\mathscr{A}$ generates the $\sigma$-algebra $\mathscr{B}$, any element of $\mathscr{B}$ may be arbitrarily closely approximated by an element of $\mathscr{A}$. In particular, there exists a finite union of cylinders $S$ such that $\mu(A \triangle S)<\delta^{2}$. Since $S$ consists of a finite union of cylinders, one of these cylinders has a maximum length and in particular, there exists a $k$ such that all of the cylinders forming $S$ have length less than $n_{k}$. We may then assume that $S$ is formed of disjoint cylinders of length exactly $n_{k}$, say $C_{1}, \ldots C_{r}$. A cylinder will be called good if it satisfies $\mu(C \backslash A) / \mu(C)<\delta$ and bad otherwise. We let $\mathscr{G}$ be the union of the good cylinders forming $S$ and let $B$ be the union of the bad cylinders forming $S$. Since the cylinders forming $B$ are disjoint and for each, we have $\mu(C) \leq \mu(C \backslash A) / \delta$, it follows that $\mu(B) \leq \mu(B \backslash A) / \delta \leq \mu(S \backslash A) / \delta<\delta$. Now, we have that $G=S \backslash B$ consists of a finite disjoint union of good $n_{k}$-cylinders. Further, we have $\mu(G \triangle A) \leq \mu(G \triangle S)+\mu(S \triangle A)<\delta+\delta^{2}<\epsilon$.

Now if $C$ is an $n_{k}$-cylinder forming part of $G$, then $C$ is one of the $n_{k}$-cylinders forming $C_{k}(b)$ for some $b \in Z$. We now show that any other cylinder making up $C_{k}(b)$ is also good. To show this, let $D$ be another $n_{k}$-cylinder which is a subset of $C_{k}(b)$. Then there exists a $\gamma \in \Gamma$ which only affects coordinates up to the $n_{k-1}$ th such that $\gamma(C)=D$. Further, restricted to $C, \gamma$ is a measure-preserving map. Since we assumed that $A$ consisted of a union of $\sim$-equivalence classes, it follows that $\mu(A \cap D)=\mu(A \cap C)$ from which it follows that $D$ is good as required.

Finally, let $\widetilde{G}$ be the union of those $C_{k}(b)$ which intersect $G$. Then from the above, it follows that $\mu(\widetilde{G} \cap A) / \mu(\widetilde{G})>1-\delta$. In particular, $\mu(\widetilde{G} \backslash A)<\epsilon$. But we have 
also that $\mu(A \backslash \widetilde{G})<\mu(A \backslash G)<\epsilon$ so we see that $\mu(A \Delta \widetilde{G})<2 \epsilon$, proving the claim that any element of $\mathscr{G}$ may be arbitrarily well approximated by unions of sets of the form $C_{k}(b)$. From this, it follows that these sets generated the $\sigma$-algebra $\mathscr{G}$.

We now show that the quotient space $Y$ may be identified with $Z$. There is a natural map from $Y$ to $Z$ and the above shows that any measurable subset of $Y$ agrees with the inverse image of a Borel measurable subset of $Z$ up to a set of measure 0 . This is sufficient to guarantee the identification of $Y$ and $Z$. We are also able to calculate the quotient measure on $Z$. This is defined by

$$
v\left([b]^{k}\right)=\frac{1}{2} \prod_{i=1}^{k}\left(\begin{array}{c}
m_{i} \\
b_{i}
\end{array}\right)\left(1-p_{i}\right)^{m_{i}-b_{i}} p_{i}^{b_{i}} .
$$

This is because the inverse under the projection of the cylinder set $[b]^{k}$ is the union of $\prod_{i=1}^{k}\left(\begin{array}{c}m_{i} \\ b_{i}\end{array}\right)$ cylinder sets in $X$ of measure $\frac{1}{2} \prod_{i=1}^{k}\left(1-p_{i}\right)^{m_{i}-b_{i}} p_{i}^{b_{i}}$.

Having identified the quotient space, define a function $\phi$ on $X$ by $\phi(x)=\min \{\log$ $d \mu \circ \gamma / d \mu(x): \log d \mu \circ \gamma / d \mu(x)>0\}$. Since the $R_{i}$ were taken to be greater than 1 , we see that this is a strictly positive quantity (and in fact bounded below by min $\left.R_{i}\right)$. Further, it is clear that if $x \sim y$, then $\phi(x)=\phi(y)$. This shows that $\phi$ may be regarded as a function on the quotient $Z$.

The final ingredient in the construction of Hamachi and Osikawa is the construction of an automorphism $U$ of $X$ such that $d \mu \circ U / d \mu=\exp \phi(x)$. Again, it is clear that if $x \sim y$ then $U(x) \sim U(y)$ so once again, $U$ may be regarded as a map of $Z$. Clearly from the construction of the measure on $X, U(x)$ should be a point in the orbit of $x$ which has the transitions modified in such a way that

$$
\frac{\mu\left([U(x)]^{n_{k}}\right)}{\mu\left([x]^{n_{k}}\right)}=\left(\frac{1-p_{1}}{p_{1}}\right)^{a_{1}-b_{1}}\left(\frac{1-p_{2}}{p_{2}}\right)^{a_{2}-b_{2}} \ldots\left(\frac{1-p_{k}}{p_{k}}\right)^{a_{k}-b_{k}}
$$

is minimal but greater than 1 , where $n_{k}$ is defined such that $U(x)$ only disagrees with $x$ before the $n_{k}$ th terms and $\left(a_{0}, a_{1}, \ldots\right)$ and $\left(b_{0}, b_{1}, \ldots\right)$ are the block types of $U(x)$ and $x$. One can identify the effect of $U$ on the cycle types. Namely, $U$ increases $b_{1}$ by 1 to $a_{1}$ unless $b_{1}$ is already maximal, in which case $a_{1}$ is set to 0 and $b_{2}$ is increased (unless $b_{2}$ should happen to be maximal and so on). This is nothing other than an odometer action where $b_{i}$ can range between 0 and $m_{i}$. This determines $U$ apart from its effect on $b_{0}$. This is determined by the requirement that $x$ and $U(x)$ should lie in the same $\Gamma$-orbit which determines that the total number of transitions up to $n_{k}$ for $x$ and $U(x)$ should have the same parity (even or odd). The digit $a_{0}$ is then a 'parity bit' which must be chosen to ensure that $a_{0}+a_{1}+\cdots+a_{k}$ differs from $b_{0}+b_{1}+\cdots+b_{k}$ by an even number.

We call the automorphism $U$ of $Z$ an odometer with parity. The ergodicity of such odometers with parity is not immediately apparent, but they turn out always to be ergodic. This will in any case follow from results about the associated flow. 
Finally, the construction of Hamachi and Osikawa gives an explicit description of the associated flow. Namely, it is isomorphic to the suspension flow of $U: Z \longrightarrow Z$ with ceiling function $\phi(z)$. To describe this, let $Z_{\phi}$ denote the space $\{(z, t): z \in$ $Z, 0 \leq t<\phi(z)\}$. The flow on this space is given by the maps $T_{s}$ where $s>0$,

$$
T_{s}((z, t))= \begin{cases}(z, s+t) & \text { if } s+t<\phi(z) \\ (U(z), s+t-\phi(z)) & \text { if } \phi(z) \leq s+t<\phi(z)+\phi(U(z)) \\ \cdots & \end{cases}
$$

Since the map $U$ is invertible, the flow is also defined for negative time. An alternative description of the flow is the following. An equivalence relation is defined on the space $Z \times \mathbb{R}$, namely $\approx$ is the equivalence relation generated by (that is, the transitive closure of $)(z, t) \approx(U(z), t-\phi(z))$. Then letting $[(z, t)]$ denote the $\approx$-equivalence class of $(z, t), T_{s}$ acts on the quotient space $Z \times \mathbb{R} / \approx$ by $T_{s}[(s, t)]=[(z, t+s)]$. In particular, we see that if $z$ and $z^{\prime}$ lie in the same orbit, then we may pick $x$ and $x^{\prime}$ which lie in the same orbit and whose block types are respectively $z$ and $z^{\prime}$ which lie on the same orbit. There is then a $\gamma_{0} \in \Gamma$ such that $\gamma_{0}(x)=x^{\prime}$. The ratio $d \mu \circ \gamma / d \mu(x)$ is determined by the block types of $x$ and $x^{\prime}$ (namely $z$ and $z^{\prime}$ ) alone so is independent of the particular values of $x$ and $x^{\prime}$. Forming $\tau=\log d \mu \circ \gamma_{0} / d \mu(x)$, we show that $(z, t) \approx\left(z^{\prime}, t-\tau\right)$. Note that in doing this, we may assume that $\tau$ is positive. Since the ratios $\log d \mu \circ \gamma / d \mu(x)$ take values in a discrete set, there can only be finitely many values of $\log d \mu \circ \gamma / d \mu$ between 0 and $\tau$ ( $j$ say). Write $\mathscr{R}(x)$ for $\{\log d \mu \circ \gamma / d \mu(x): \gamma \in \Gamma\}$. Then by the chain rule, we see that $\mathscr{R}(\gamma(x))=\mathscr{R}(x)-\log d \mu \circ \gamma / d \mu(x)$. It now follows that $U^{j}(z) \sim z^{\prime}$. Now we see $\tau=\phi(z)+\phi(U(z))+\cdots+\phi\left(U^{j-1}(z)\right)$. In particular, we have that $(z, t) \approx\left(z^{\prime}, t-\tau\right)$. This is extremely important as it shows that $T_{\tau}(z, t)=\left(z^{\prime}, t\right)$. Letting $K_{j}$ denote $\log \left(\left(1-p_{j}\right) / p_{j}\right)$, we see that in the case where $z_{j}<m_{j}, T_{K_{j}}(z, t)=(\widehat{z}, t)$, where $\widehat{z}_{i}=z_{i}+\delta_{i j}$.

It remains to demonstrate that this flow has the AT property. We will let $\chi_{s}$ denote the characteristic function of a set $S$ and use the notation $[z]^{k}$ or $\left[b_{0} \ldots b_{k}\right]$ for cylinder sets in $Z$. Fix $k>0$. Then we will show, taking sufficiently small $\delta$ and letting $f=\chi_{[0 \ldots . .0]^{k} \times[0 . \delta]}$, that we can closely approximate arbitrarily closely any given finite collection of functions of the form $\chi_{\left[b_{0} \ldots b_{k}\right]^{k} \times[c . d]}$. Since any positive integrable function may be arbitrarily closely approximated by a finite linear combination of functions of this form, it will follow from this that the flow has the AT property.

First, we observe that letting $\tau=\sum_{i=1}^{k} a_{i} K_{i}$ where $0 \leq a_{i} \leq m_{i}$, we have

$$
\mathscr{L}_{-\tau} f(z, t)=\chi_{[00 \ldots 0)^{k} \times[0 . \delta]}\left(T_{-\tau}(z, t)\right) \frac{d \mu \circ T_{-\tau}}{d \mu}(z, t) .
$$

But we see that $\chi_{[00 \ldots 0\}^{k} \times[0 . \delta]}\left(T_{-\tau}(z, t)\right)$ is equal to $\chi_{T_{\mathrm{r}}\left([00 \ldots .0\}^{k} \times[0 . \delta]\right)}(z, t)$ and it is straightforward to check that $T_{\tau}\left([00 \ldots 0]^{k} \times[0, \delta]\right)=\left[c(a) a_{1} a_{2} \ldots a_{k}\right]$ where $c(a)$ is such 
that $c(a)+a_{1}+\ldots+a_{k}=0(\bmod 2)$. This follows from the fact that if $x$ has block type in $[00 \ldots 0]^{k}$ and $\gamma \in \Gamma$ affect only the first $n_{k}$ coordinates, leaving $\gamma(x) \in\left[c(a) a_{1} a_{2} \ldots a_{k}\right]$ then $\log d \mu \circ \gamma / d \mu(x)=\tau$. This means that $\mathscr{L}_{-\tau} f$ is just

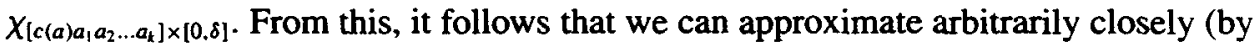
taking small $\delta)$ any function of the form $\chi_{[b]^{k} \times[c, d]}$ provided that $\sum_{i=0}^{k} b_{i}=0(\bmod 2)$. It remains to show that we can approximate cylinders where $\sum_{i=0}^{k} b_{i}=1(\bmod 2)$.

To this end, pick a very large $M$ and consider $\mathscr{L}_{-K_{M}} f$. Then as before, we have $f\left(T_{-K_{M}}(z, t)\right)=\chi_{r_{K_{M}}\left([0 \ldots o)^{k} \times[0 . \delta]\right)}(z, t)$. Then we observe that if $(y, s) \in[0 \ldots 0]^{k} \times$ $[0, \delta]$ and $y_{M} \neq m_{M}$ then $T_{K_{M}}(y, s)=(\widehat{y}, s)$ where $\widehat{y}_{0}=1$ and $\widehat{y}_{i}=y_{i}+\delta_{i M}$ for $i \geq 1$. Setting $B=\left\{(z, t) \in[0 \ldots 0]^{k} \times[0, \delta]: z_{M} \neq m_{M}\right\}$, and $B^{\prime}=\left([0 \ldots 0]^{k} \times[0, \delta]\right) \backslash B$,

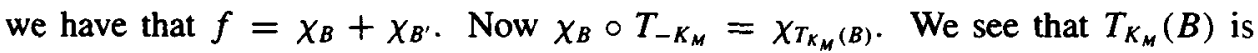
equal to $S=\left\{(z, t) \in[100 \ldots 0]^{k} \times[0, \delta]: z_{M} \neq 0\right\}$. It follows that $\mathscr{L}_{-K_{M}} \chi_{B}$ is equal to $C \chi_{S}$ where $C$ is a constant. Since $\left\|\chi_{B^{\prime}}\right\|_{1}=p_{M}{ }^{m_{M}}\|f\|_{1}$ and $\mathscr{L}_{-K_{M}}$ is linear and norm-preserving, we have

$$
\begin{aligned}
\left\|\mathscr{L}_{-K_{M}} f-C \chi_{[100 \ldots 0]^{k} \times[0 . \delta]}\right\|_{1} & \leq\left\|\mathscr{L}_{-K_{M}} \chi_{B}-C \chi_{[100 \ldots 0)^{k} \times[0 . \delta]}\right\|_{1}+\left\|\chi_{B^{\prime}}\right\|_{1} \\
& \leq\left(p_{M}{ }^{m_{M}}+\left(1-p_{M}\right)^{m_{M}}\right)\|f\|_{1} .
\end{aligned}
$$

This shows that for large $M$, we can get an arbitrarily close approximation to $\chi_{[100 \ldots 0]^{k} \times[0, \delta]}$ and then by a similar argument to that for the even parity cylinders, we see that we can approximate any function of the desired type. This completes the proof.

\section{References}

[1] G. Brown and A. H. Dooley, 'Ergodic measures are of weak product type', Math. Proc. Cambridge Philos. Soc. 98 (1985), 129-145.

[2] G. Brown and A. H. Dooley, 'Odometer actions on $G$-measures', Ergodic Theory Dynamical Systems 11 (1991), 279-307.

[3] G. Brown, A. H. Dooley and J. Lake, 'On the Krieger-Araki-Woods ratio set', Tôhoku Math. J. 47 (1995), 1-13.

[4] J. R. Choksi, J. M. Hawkins and V. S. Prasad, 'Abelian cocycles for nonsingular ergodic transformations and the genericity of type III $_{1}$ transformations', Monatsh. Math. 103 (1987), 187-205.

[5] A. Connes and E. J. Woods, 'Approximately transitive flows and ITPFI factors', Ergodic Theory Dynamical Systems 5 (1985), 203-236.

[6] K. L. Chung A course in probability theory (Academic Press, New York, 1974).

[7] A. H. Dooley and T. Hamachi, 'Markov odometer actions not of product type', Trans. Amer. Math. Soc., to appear.

[8] H. Dye, 'On groups of measure preserving transformations II', Amer. J. Math. 95 (1963), 551-576.

[9] T. Hamachi and M. Osikawa, 'Computation of the associated flows of ITPFI factors of type $I I I_{0}$ ', in: Geometric methods in operator algebras (eds. H. Araki and E. G. Effros) Pitman Research Notes 123 (Longman, New York, 1986), pp. 126-210. 
[10] T. Hamachi, 'A measure-theoretical proof of the Connes-Woods theorem on AT-flows', Pacific J. Math. 154 (1992), 67-85.

[11] T. Hamachi, Y. Oka and M. Osikawa, 'A classification of ergodic non-singular transformation groups', Mem. Fac. Sci., Kyushu Univ. Ser. A 28 (1974), 115-133.

[12] J. M. Hawkins, 'Properties of ergodic flows associated to product odometers', Pacific J. Math. 141 (1990), 287-294.

[13] W. Krieger, 'On non-singular transformations of a measure space I', Z Wahrscheinlichkeitstheorie verw. Geb. 11 (1969), 83-91; II, Z. Wahrscheinlichkeitstheorie verw. Geb. 11 (1969), 98-1 19.

[14] W. Krieger, 'On the Araki-Woods asymptotic ratio set and non-singular transformations of a measure space', Lecture Notes in Math. 160 (Springer, Berlin, 1970), pp. 158-177.

[15] W. Krieger, 'On the infinite product construction of non-singular transformations of measure space', Invent. Math. 15 (1972), 144-163.

[16] C. C. Moore, 'Invariant measures on product spaces', Proceedings of the fift Berkeley symposium, (1967), 447-459.

[17] C. E. Sutherland, Notes on orbit equivalence, Lecture note series 23 (Univ. i Oslo, 1976).

School of Mathematics

University of New South Wales

Sydney 2052

Australia

e-mail: a.dooley@unsw.edu.au

Department of Mathematical Sciences

University of Memphis

Memphis, TN 38152

USA

e-mail:quasa@msci.memphis.edu
Department of Mathematics and Statistics

McGill University 805 Sherbrooke St. W.

Montreal, Quebec

Canada H3A 2K6

e-mail: klemes@math.mcgill.ca 